PHYSICAL REVIEW D 90, 123532 (2014)

\title{
Potential of the Large Observatory for X-ray Timing telescope for the search for dark matter
}

\author{
A. Neronov, ${ }^{1}$ A. Boyarsky, ${ }^{2,3,4}$ D. Iakubovskyi, ${ }^{4,5}$ and O. Ruchayskiy ${ }^{6,3}$ \\ ${ }^{1}$ Department of Astronomy, ISDC Data Centre for Astrophysics, University of Geneva, \\ Chemin d'Ecogia 16, 1290 Versoix, Switzerland \\ ${ }^{2}$ Instituut-Lorentz for Theoretical Physics, Universiteit Leiden, Niels Bohrweg 2, Leiden, Netherlands \\ ${ }^{3}$ Ecole Polytechnique Fédérale de Lausanne, CH-1015 Lausanne, Switzerland \\ ${ }^{4}$ Bogolyubov Institute of Theoretical Physics, Metrologichna Street 14-b, 03680 Kyiv, Ukraine \\ ${ }^{5}$ National University "Kyiv-Mohyla Academy," Skovorody Street 2, 04070 Kyiv, Ukraine \\ ${ }^{6}$ CERN Physics Department, Theory Division, CH-1211 Geneva 23, Switzerland
}

(Received 18 December 2013; published 24 December 2014)

\begin{abstract}
Large observatory for x-ray timing (LOFT) is a concept of a next-generation $\mathrm{x}$-ray telescope considered in the context of the "Cosmic Vision" program of the European Space Agency. The Large Area Detector on board of LOFT will be a collimator-type telescope with an unprecedentedly large collecting area of about $10^{5} \mathrm{~cm}^{2}$ in the energy band between 2 and $100 \mathrm{keV}$. We demonstrate that LOFT will be a powerful dark matter detector, suitable for the search of the x-ray line emission expected from decays of light dark matter particles in galactic halos. We show that LOFT will have sensitivity for dark matter line search more than an order of magnitude higher than that of all existing x-ray telescopes. In this way, LOFT will be able to provide a new insight into the fundamental problem of the nature of dark matter.
\end{abstract}

DOI: 10.1103/PhysRevD.90.123532

PACS numbers: 95.35.+d, 95.55.Ka

\section{INTRODUCTION}

The nature of dark matter $(\mathrm{DM})$ is one of the most intriguing questions of modern physics. Mass content of galaxies and galaxy clusters, growth of density fluctuations through the cosmic history, large scale structure of the Universe-all point towards the existence of new substance, the DM, which constitutes some $80 \%$ of the total mass content of the Universe. If DM is made of particles, these particles are not among the known ones. Phenomenologically little is known about properties of DM particles:

(i) Their overall density is $\Omega_{\mathrm{DM}} h^{2}=0.1196 \pm 0.0031$;

(ii) the mass of any fermionic DM is limited from below by the "Tremaine-Gunn bound" [1], while for bosons such a limit is significantly lower $[2,3]$.

(iii) Dark matter particles are not necessarily stable, but their lifetime should significantly exceed the age of the Universe (see e.g. [4-6]);

(iv) DM particles should have become nonrelativistic sufficiently early in the radiation-dominated epoch (although a subdominant fraction might have remained relativistic much later [7]).

Depending on the nature of interaction of DM particles with ordinary matter today, the DM can have different astrophysical signatures (see e.g. [8,9]). Two main classes of DM particle candidates are considered: annihilating and decaying.

A lot of attention has been devoted to a class of annihilating DM candidates called weakly interacting massive particles (WIMPs) (see e.g. [10,11] for review). These hypothetical particles are assumed to interact with ordinary matter with roughly electroweak strength and have masses in $\mathcal{O}\left(1-10^{3}\right) \mathrm{GeV}$ to provide the correct DM abundance. Due to their large mass and interaction strength these particles should be stable (i.e. their decays should be prohibited by a conservation law, otherwise their lifetime would be too short). Astrophysical signature of their annihilation products is an important scientific goal of many cosmic missions $[9,12]$. In particular, $\gamma$ rays from DM annihilation are extensively searched with $\gamma$-ray telescopes $[13,14]$.

There is a large class of DM candidates that interacts with the ordinary particles superweakly (i.e. significantly weaker than neutrinos). These include extensions of the standard model (SM) by right-hand neutrinos [15-17], models with extra dimensions and string-motivated models [18], gravitinos [19,20], axions [21,22], and axinos [23,24] (see e.g. $[11,25,26]$ for reviews). These candidates are as possible as WIMPs and from many points of view are very compelling. The feeble interaction strength of these DM candidates means that unlike WIMPs (i) their mass is not restricted to the $\mathrm{GeV}$ region; and (ii) they can decay into the SM particles. The fermionic DM candidates (such as sterile neutrino, gravitino, axino) possess a 2-body radiative decay channel, DM $\rightarrow \gamma+\nu$, while bosonic DM candidates (such as e.g. axion or Majoron) can decay into two photons. These 2-body decays produce photons with energy $E_{\gamma}=\frac{1}{2} M_{\mathrm{DM}} c^{2}$. The cosmologically long lifetime makes the intrinsic width of such a line negligible. This provides a clear observational signature of decaying DM candidates: a narrow spectral line in spectra of large DM concentrations, such as galaxies and galaxy clusters. 
Search of the DM decay signal in the $\mathrm{keV}-\mathrm{MeV}$ mass range was conducted using XMM-Newton [27-33], Chandra [34-39], Suzaku [40,41], Swift [42], INTEGRAL [43,44] and HEAO-1 [27] telescopes, as well as a rocket-borne $\mathrm{x}$-ray microcalorimeter [45]. Observations of extragalactic diffuse x-ray background [27,46]; galaxy clusters $[28,35,36]$; Milky Way, Andromeda (M31) and Triangle (M33) galaxies [28-31,43,44,46]; and dwarf spheroidal satellites of the Milky Way [29,33,37,39-41,45] allowed us to put important constraints on parameters of decaying DM particles. Lower bounds on decaying DM lifetime, derived from the $\mathrm{x}$-ray and $\gamma$-ray data are at least eight orders of magnitude longer than the age of the Universe [4] (see also [6] for extension to higher energies). Table I summarizes existing works that put bounds on decaying DM from observations of individual objects. In this table, we do not mention the claim [47] that the intensity of the Fe XXVI Lyman- $\gamma$ line at $8.7 \mathrm{keV}$, observed in [48], cannot be explained by standard ionization and recombination processes, and that the dark matter decay may be a possible explanation of this apparent excess. Spectral resolution of current missions does not allow us to reach any conclusion. However, barring an exact coincidence between energy of decay photon and Fe XXVI Lyman- $\gamma$, this claim may be tested with the new missions, discussed in e.g. [49].

In what follows we argue that a next-generation x-ray mission Large Observatory for X-ray Timing (LOFT) will provide a crucial improvement in the sensitivity for the search of decaying DM in x-rays. LOFT mission was studied at the European Space Agency (ESA) as one of the five medium mission candidates for the "M3" mission in the framework of the Cosmic Vision program of ESA. It will be reproposed as a candidate for the "M4" mission within the same program. Further details on the LOFT mission could be found in [55]. Although the search for the decaying DM is not listed among the primary scientific goals of LOFT, we show that LOFT will have a capability to explore almost the entire parameter space of one of the most often discussed models of decaying DM, the neutrino minimal extension of the standard model of particle physics ( $\nu$ MSM) [16]. This will be possible if LOFT will be converted into a dedicated DM detection experiment (e.g. toward the end of the mission) aimed at ultradeep exposure of the most favorable (massive, relatively compact) nearby DM halo.

TABLE I. Summary of existing x-ray observations of different objects performed by different groups. The characteristic sizes of

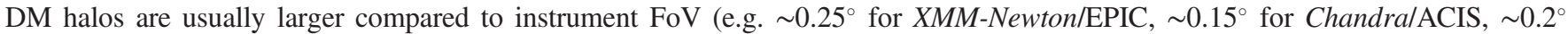
for Suzaku/XIS).

\begin{tabular}{|c|c|c|c|c|}
\hline Reference & Object & Instrument & Cleaned exp, ks & $\begin{array}{l}\text { Characteristic } \\
\text { halo size, deg }\end{array}$ \\
\hline [27] & Diffuse x-ray background & HEAO-1, XMM-Newton & 224,1450 & $\lesssim 180$ \\
\hline [28] & Coma and Virgo galaxy clusters & XMM-Newton & 20,40 & $0.2-0.5, \sim 2$ \\
\hline [29] & Large Magellanic Cloud & XMM-Newton & 20 & $\sim 10$ \\
\hline [34] & Milky Way halo & Chandra/ACIS-S3 & Not specified & $\lesssim 180$ \\
\hline$[30]$ & M31 (central 5') & XMM-Newton & 35 & $1-3$ \\
\hline$[35]$ & Abell 520 galaxy cluster & ChandralACIS-S3 & 67 & $\sim 0.1$ \\
\hline [31] & Milky Way halo, Ursa Minor dSph & $X M M-N e w t o n$ & 547,7 & $\lesssim 180$ \\
\hline [46] & Milky Way halo & Chandra/ACIS & 1500 & $\lesssim 180$ \\
\hline [36] & Galaxy cluster 1E 0657-56 (“bullet”) & Chandra/ACIS-I & 450 & $\sim 0.1$ \\
\hline [45] & Milky Way halo & $\mathrm{X}$-ray microcalorimeter & 0.1 & $\lesssim 180$ \\
\hline [43] & Milky Way halo & INTEGRAL/SPI & 5500 & $\lesssim 180$ \\
\hline$[32]$ & M31 (central 5-13') & XMM-Newton/EPIC & 130 & $1-3$ \\
\hline [44] & Milky Way halo & INTEGRAL/SPI & 12200 & $\lesssim 180$ \\
\hline [40] & Ursa Minor & Suzaku/XIS & 70 & $\sim 1$ \\
\hline [37] & Draco dSph & Chandra/ACIS-S & 32 & $\sim 0.5$ \\
\hline [38] & Willman 1 & Chandra/ACIS-I & 100 & $\sim 0.1$ \\
\hline [39] & M31, Fornax, Sculptor & XMM-Newton/EPIC, Chandra/ACIS & $400,50,162$ & $1-3,0.2-1.0,0.5-1.0$ \\
\hline$[50]$ & Willman 1 & Chandra/ACIS-I & 100 & $\sim 0.1$ \\
\hline [42] & Segue 1 & Swift/XRT & 5 & $\sim 0.3$ \\
\hline$[51]$ & M33 & XMM-Newton/EPIC & $20-30$ & $\sim 0.5$ \\
\hline$[52]$ & M31 (12-28' off-center) & Chandra/ACIS-I & 53 & $1-3$ \\
\hline [33] & Willman 1 & XMM-Newton & 60 & $\sim 0.1$ \\
\hline [41] & Ursa Minor, Draco & Suzaku/XIS & 200,200 & $\sim 1, \sim 0.5$ \\
\hline [53] & M31 (2-16' off-center) & Chandra/ACIS-I & 404 & $1-3$ \\
\hline [54] & stacked dSphs & XMM-Newton/EPIC & 405 & $0.1-0.3$ \\
\hline
\end{tabular}




\section{STRATEGY OF SEARCH FOR DECAYING DARK MATTER}

The number of photons from DM decay detectable by a telescope is proportional to the DM column density $S_{\mathrm{DM}}=\int \rho_{\mathrm{DM}}(r) d r$ integrated along the line of sight. As it turns out, this signal is very weakly dependent on the virial mass of the DM halo and on the assumed dark matter density profile $[29,56,57]$. Moreover, for objects that cover the whole field of view of the telescope, the expected DM decay flux is independent of the distance to the object. As a result a vast variety of DM-dominated objects (nearby galaxies and galaxy clusters) produce a comparable decay signal. Therefore (i) one has a freedom of choosing the observational targets, avoiding complicated astrophysical backgrounds; (ii) if a candidate line is found, its surface brightness profile may be measured, distinguished from known atomic lines and compared among several objects with the same expected signal (see e.g. [39]). This allows us to distinguish the decaying DM line from astrophysical backgrounds; see [54,58,59] for a recent discussion of a candidate DM decay line at $3.5 \mathrm{keV}$. The case of the astrophysical search for decaying DM has been presented in the recent white papers $[60,61]$.

With intrinsic width of the decay line being negligible, its broadening is determined entirely by the virial velocity of DM particles, confined in the DM halo: $E / \Delta E \simeq c / v_{\text {vir }}$. This number ranges from $10^{2}$ for galaxy clusters to $10^{4}$ for dwarf spheroidal galaxies. The spectral resolution of modern x-ray instruments is insufficient for the measurement of the line width (with an exception of Spectrometer on INTEGRAL (SPI) instrument, see [44]). The narrow line is detected on top of a continuum background. This background has two main contributions - astrophysical and instrumental. The astrophysical background is a continuum thermal and nonthermal emission from the astrophysical source, plus the $\mathrm{x}$-ray emission from the local interstellar medium in the Milky Way plus the cosmic x-ray background (CXB) [62] within the instrument's field of view (FoV). The instrumental background is produced by the charged particles passing through the detector and by the electronics noise. The line signal is centered on the reference line energy $E$ and is smeared over the energy range $\sim \Delta E$ where $\Delta E$ is the spectral resolution of photon detector. The amount of background accumulated in this energy bin is proportional to the bin width $\Delta E$. Thus, improvement of the energy resolution results in the decrease of the background and, as a consequence, improvement of the sensitivity of the instrument for the line detection.

The line signal increases with the increase of collection area of the detector. For an extended source, is also increases with the extension of the telescope FoV. Overall, the signal is proportional to the product of the effective area, $A_{\text {eff }}$, and the solid angle subtended by the FoV (for those DM halos that have angular size larger than the FoV) that is to the "grasp" $A_{\text {eff }} \Omega_{\text {fov }}$ of the instrument
[45]. Comparison of potential of different instruments for the detection of DM decay line could be conveniently presented in terms of "energy resolution vs grasp" diagram [45], as shown in Fig. 1.

In this figure, the inclined lines show the "equal sensitivity" sets of instrumental characteristics. Indeed, the signal-to-noise ratio for the DM decay line sensitivity improves as $R \propto \sqrt{A_{\text {eff }} \Omega_{\text {fov }} / \Delta E}$, so that the lines grasp $\propto$ energy resolution correspond to instruments which provide the same signal-to-noise ratio if they operate in the same energy band. One could define $R$ as a "figure of merit" for the weak line search; see e.g. [63]. We have arbitrarily fixed $R=1$ for the parameter choice corresponding to the averaged over the energy band characteristics of the EPIC camera of the XMM-Newton telescope [45].

The comparison shown in Fig. 1 adopts an assumption that the level of background in different instruments is comparable. This is true if the background on top of which the DM signal is searched is the CXB. However, if the

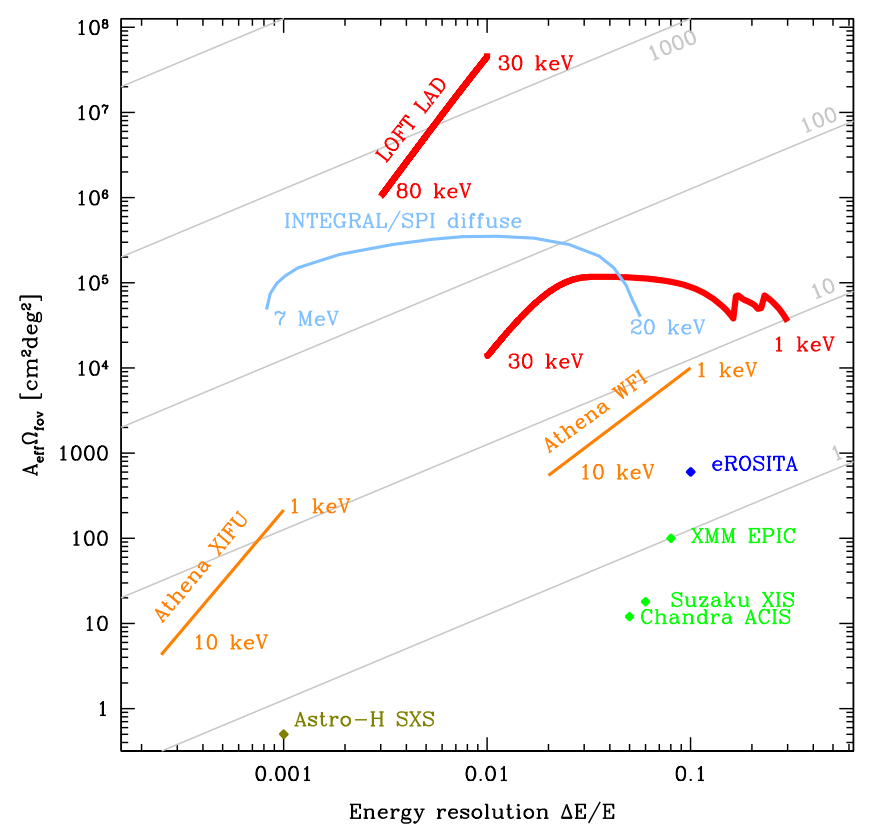

FIG. 1 (color online). Sensitivity of x-ray telescopes for the dark matter decay line detection in terms of the energy resolution vs grasp diagram (c.f. [45]). Two red solid curves correspond to the Large Area Detector (LAD) (in the "requirements" configuration) in two different observation modes: observations of localized sources of the angular extent $\mathrm{i} \gtrsim 1^{\circ}$ range and observations of the large angular scale diffuse emission from the Milky Way with the steradian-sized FoV of LAD at higher energies. Inclined grey lines with marks in 1-100 range show improvement of the sensitivity for the line search due to the increase of effective area / FoV and improvement of energy resolution. Level "1" corresponds to average parameters of the XMM-Newton EPIC camera. Other curves with labels show the improvements of sensitivity with the named instruments (Astro-H, Athena, eROSITA etc.) in their respective energy ranges (marked where possible). 


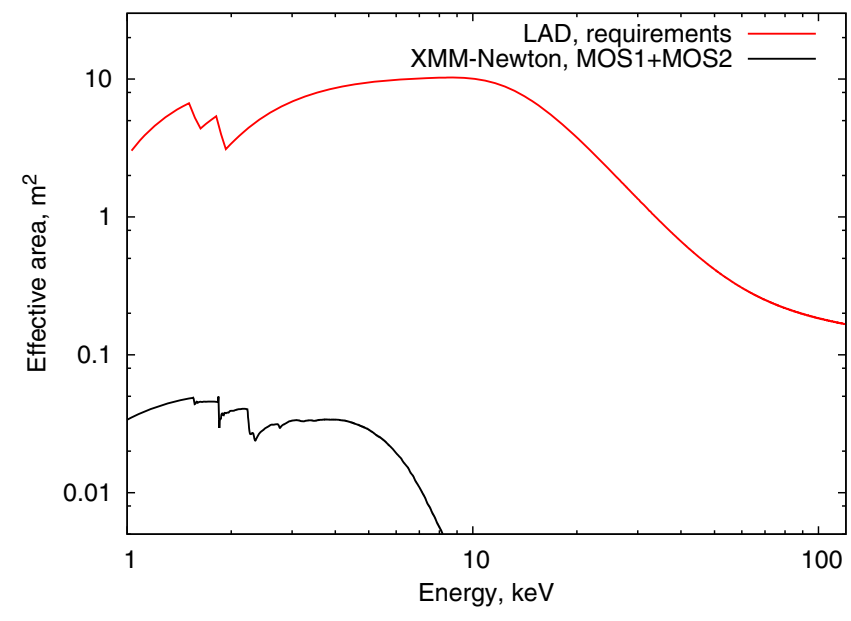

FIG. 2 (color online). Effective area for LAD instrument in the requirements payload. For comparison the effective area of combined EPIC MOS1 + MOS2 cameras of XMM-Newton is shown in black.

background is of instrumental nature, the comparison of different instruments has to include an additional parameter, which is the level of background. We include this parameter in our considerations below.

\section{LOFT CHARACTERISTICS RELEVANT FOR DM DETECTION}

The main instrument on board of LOFT will be the LAD. LAD will be an $\mathrm{x}$-ray telescope with effective collection area $A_{\text {eff }} \simeq 10 \mathrm{~m}^{2}$ (see Fig. 2) sensitive in the $2-80 \mathrm{keV}$ energy range [64]. LAD will be composed of the Silicon Drift Detectors (SDD) with energy resolution below $300 \mathrm{eV}$. The SDDs will be covered by microchannel plate collimators providing the field of view of $1^{\circ}$ in the energy range below $\simeq 30 \mathrm{keV}$ and becoming increasingly transparent to $\mathrm{x}$ rays at higher energies up to $80 \mathrm{keV}[64,65]$.

The energy resolution of LAD is determined by the characteristics of the silicon detectors and of the detector electronics [64]. Using the response functions of the LOFT satellite [66], we simulated narrow line at different energies and then approximated the obtained spectrum by the Gaussian profile (see left panel of Fig. 3). The obtained best-fit value of Gaussian dispersion is then used to calculate FWHM. The results are shown in the right in Fig. 3. They can be approximated as a linear function of energy:

$$
\operatorname{FWHM}(E)=0.213 \mathrm{keV}+4.10 \times 10^{-3} \frac{E}{\mathrm{keV}} .
$$

Our analysis considers two possible LOFT configurations [55]: requirements and "goal." Parameters of each configuration are summarized in Table II.
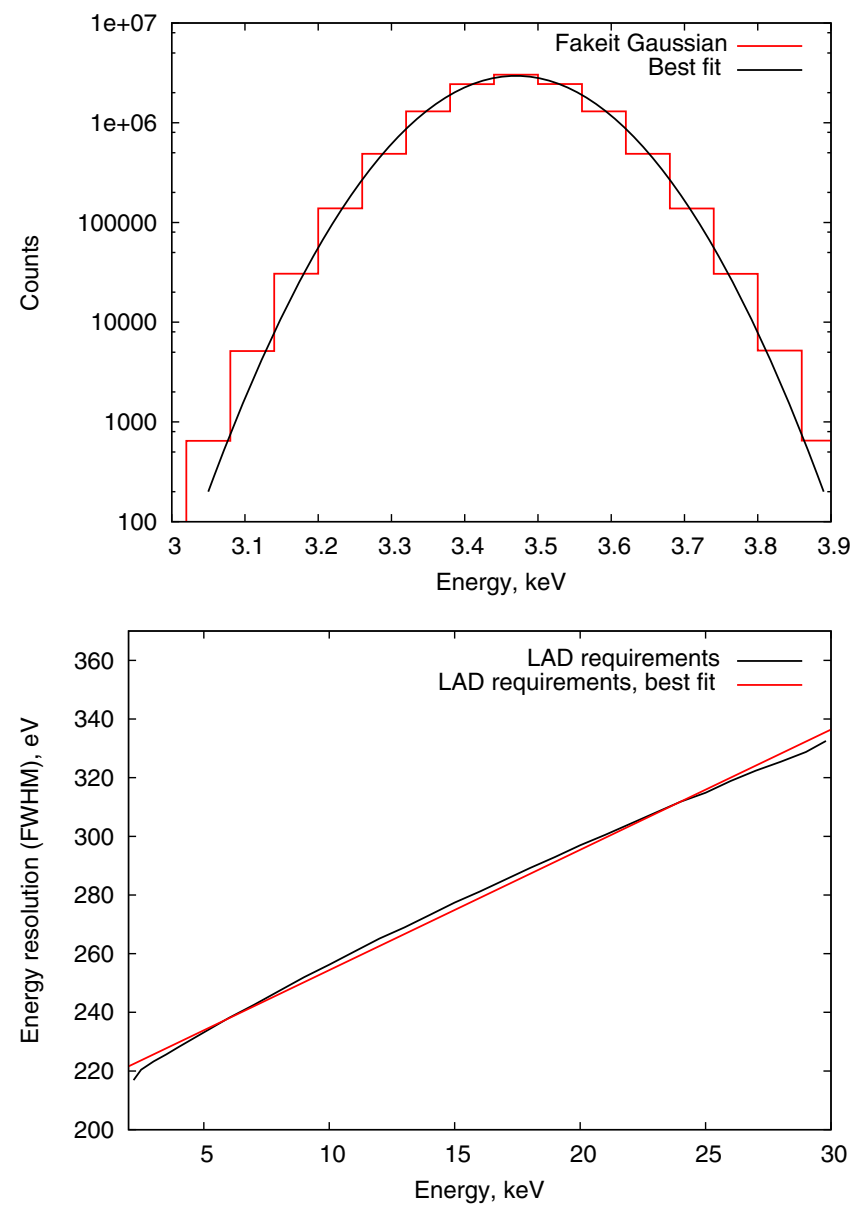

FIG. 3 (color online). Up: an example of simulated line at $3.5 \mathrm{keV}$, together with its best-fit Gaussian model used to calculate FWHM. Down: LAD energy resolution for requirements payload as function of energy and its best fit (1) calculated from our simulations.

\section{SENSITIVITY FOR THE DM LINE DETECTION}

\section{A. Signal from extended sources in the field of view of collimator}

We begin with an estimate of the sensitivity of the LAD detector for weak diffuse lines in the energy range below $30 \mathrm{keV}$ where the collimator limits the FoV to $1^{\circ}$. To this end we take the background spectrum shown in Fig. 4, and compute the number of background photons in the bin with the size equal to FWHM over the time $T_{\exp }$ chosen to be $100 \mathrm{ksec}$ (a typical time scale of a single observation). We then estimate the $3 \sigma$ upper limits on the line flux in each narrow energy, based on the statistical error on the background counts:

$$
F_{\text {line }, 3 \sigma}(E)<\frac{3 \sqrt{2 \times N_{\mathrm{bkg}}(E)}}{A_{\text {eff }}(E) T}
$$

(an additional $\sqrt{2}$ was included, because we assumed that we are subtracting observations from a background with comparable statistics). 
TABLE II. Scientific requirements for the LOFT LAD instrument (from $[64,65]$ ). The energy range of LOFT LAD detector can be extended beyond $30 \mathrm{keV}$ (the nominal range) to the energies up to $80 \mathrm{keV}$ (see [65] for the latter number). At those higher energies the LAD collimator becomes more and more transparent to $\mathrm{x}$ rays [64].

\begin{tabular}{lll}
\hline \hline Parameter & \multicolumn{1}{c}{ Requirements } & \multicolumn{1}{c}{ Goal } \\
\hline Energy range & $2-30 \mathrm{keV}$ & $1-40 \mathrm{keV}$ \\
& $2-80 \mathrm{keV}[64,65]$ & $1-80 \mathrm{keV}[65]$ \\
Effective area & $12.0 \mathrm{~m}^{2}(2-10 \mathrm{keV})$ & $15 \mathrm{~m}^{2}(2-10 \mathrm{keV})$ \\
& $1.3 \mathrm{~m}^{2}(@ 30 \mathrm{keV})$ & $2.5 \mathrm{~m}^{2}(@ 30 \mathrm{keV})$ \\
$\Delta \mathrm{E}$ & $<260 \mathrm{eV}$ & $<180 \mathrm{eV}$ \\
$(\mathrm{FWHM}, @ 6 \mathrm{keV})$ & & \\
FoV $(\mathrm{FWHM})$ & $<60$ arcmin & $<30$ arcmin \\
\hline \hline
\end{tabular}

The upper limit calculated in this way is shown in Fig. 5. One could see that this limit is better than that derived from an XMM-Newton exposure of the same duration. This demonstrates that in spite of somewhat higher background level of the LAD detector (contrary to XMM-Newton it includes the CXB scattered by the collimator walls [68]), the upper limit on the line flux within the FoV is better. The obvious reason for this is much larger effective area of the detector. Further improvement of sensitivity of LAD, compared to XMM-Newton (not reflected by the figure) is that LAD collects larger DM line signal in a similar exposure. This is due to the larger FoV.

\section{B. Signal from the Milky Way halo visible for a "bare detector"}

At energies above $20-40 \mathrm{keV}$, the collimator of the LAD will not be able to stop photons falling at large incidence

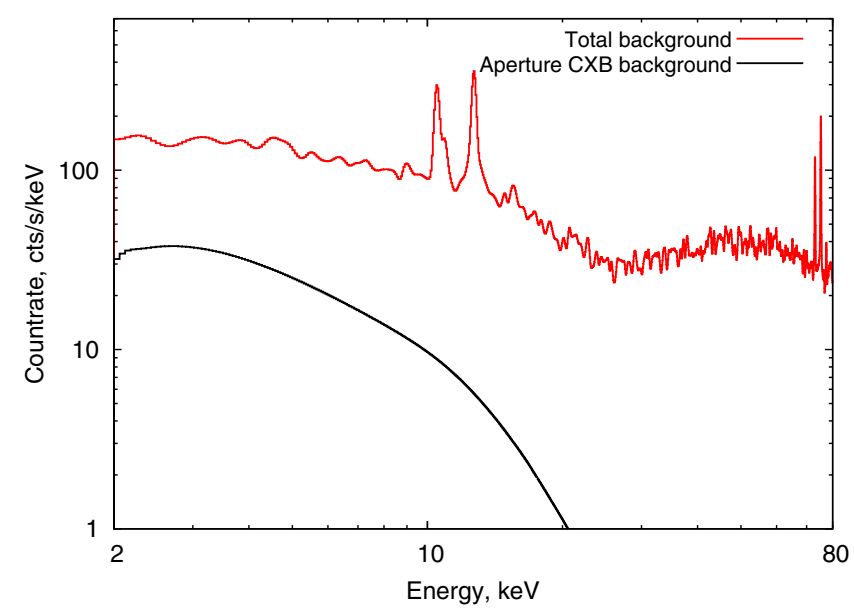

FIG. 4 (color online). Background of LAD instrument (compared to the CXB, lower curve); see also Fig. 17 of [68]. The instrumental component has been obtained using LAD_REQUIREMENTS_v6.3.BKG background file from ISDC LAD response and background page [67].

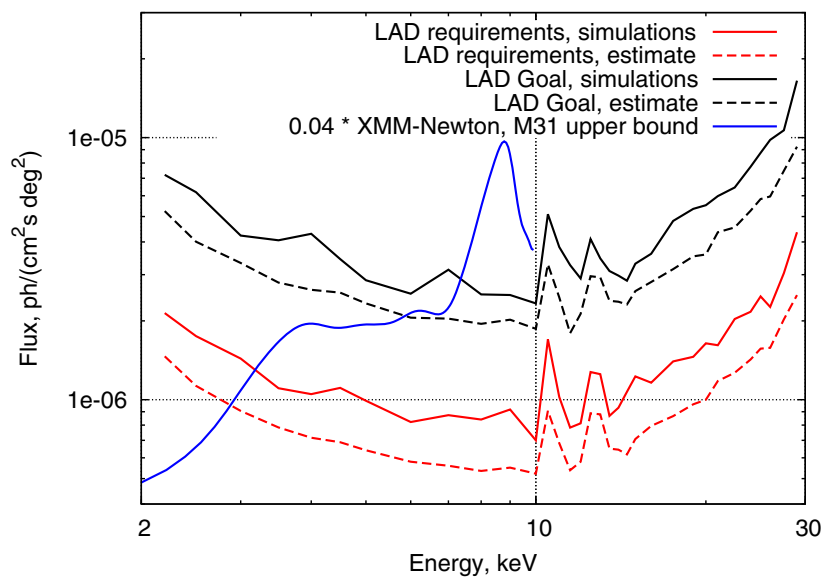

FIG. 5 (color online). The $3 \sigma$ upper bound on the flux per unit solid angle in the line from a diffuse source detectable by LAD detector. Thick lines: results based on simulations and subsequent detection of a line. Dashed line: $3 \sigma$ estimates, based on the statistical $3 \sigma$ upper bound of the instrumental background; see Eq. (2). Due to four times smaller FoV, the bound from goal payload is much weaker than from requirements payload. Rescaled $3 \sigma$ upper bound on DM flux from XMM-Newton observations of M31 central part [32] (blue) is shown for comparison.

angle, so that LAD increasingly becomes a "naked detector" sampling photons from large, steradian scale FoV. Such a design is optimal for the search of diffuse emission from the Milky Way halo [45,61]. The DM signal is accumulated in all the pointings of the telescope, no matter where the pointing is directed. This allows us to achieve extremely long exposures in a multiyear operation of the telescope. It is not possible to estimate what will be the effective field of view of the LAD detector at these energies. As an estimate we take $\Omega_{\text {fov,high }}=1 \mathrm{sr}$. We remind the reader that the sensitivity estimate, $R$, scales as $R \propto \sqrt{\Omega_{\text {fov,high }} / 1 \mathrm{sr}}$.

In the case of an all-sky source, it is a challenge to distinguish the real source signal from an instrumental feature, such as the instrumental atomic or nuclear line, which is also expected to appear in all pointings. However, a clear observational signature of the real DM decay signal is the excess toward the Galactic center (GC). This signature is readily identifiable and could be used to discriminate the real signal from the instrumental noise. This approach was used in [44]. The authors of this reference were able to identify for example the $511 \mathrm{keV}$ from the positron annihilation in the Galactic center region. It was also demonstrated that no other (instrumental) line present in the all-sky exposure has surface brightness profile (as a function of off GC angle) expected for DM decay line. This allowed the authors of [44] to derive constraints on the DM line flux in the $20 \mathrm{keV}-7 \mathrm{MeV}$ energy range using the SPI instrument of INTEGRAL as a wide-field (steradian FoV) detector. 
The same approach could be adopted to the LAD data above $\sim 20-40 \mathrm{keV}$ where the instrument works as a wide FoV detector. The main difference with the calculations of the previous section is that the central part of the Milky Way is a bright $\mathrm{x}$-ray source. The emission from this source is the sum of emission from high mass and low mass $\mathrm{x}$-ray binaries and cataclysmic variables. Measurement of the collective emission from the Milky Way sources within a steradian scale FoV by SPI [69] provides a reference value for the level of sky background on top of which the DM line signal from the Milky Way should be detected,

$$
F_{\mathrm{MW}} \simeq 10^{-4}\left(\frac{E}{100 \mathrm{keV}}\right)^{-2.5} \frac{\mathrm{ph}}{\mathrm{cm}^{2} \mathrm{~s} \mathrm{keV}} .
$$

The limits calculated for the background level (3) and a year-long exposure time are shown in Fig. 6. For comparison, the same figure shows the upper limit on the line flux within a steradian FoV of SPI found by [44]. One could see that, in accordance with the expectations, the limits which would be derived from the LAD data are tighter than those from the SPI.

\section{Limits on the decaying DM lifetime}

To convert the limits on the line flux into the limits on the lifetime of the decaying DM, $\tau_{\mathrm{DM}}$, we note that flux in line (in photons per $\mathrm{cm}^{2}$ per $\mathrm{sec}$ ) is given by

$$
F_{\text {line }}=\left(\frac{1}{\tau_{\mathrm{DM}} m_{\mathrm{DM}}}\right)\left(\frac{M_{\mathrm{fov}}}{4 \pi D_{L}^{2}}\right)
$$

where the first term is determined by the basic properties of DM particles, while the second one is the characteristic of the object being observed.

For nearby objects that cover the whole FoV of the instrument one can express

$$
\frac{M_{\mathrm{fov}}}{4 \pi D_{L}^{2}} \simeq \frac{S_{\mathrm{DM}} \Omega_{\mathrm{fov}}}{4 \pi}
$$

where $S_{\mathrm{DM}}$ is the average DM column density in a given direction. This quantity changes very little among objects of different masses and sizes [29,56,57] and its typical values are $10^{1.5 \div 3} \mathrm{M}_{\odot} / \mathrm{pc}^{2}$. Using this fact and taking into account that for 2-body decays the mass of DM is related to the energy of emitted photon via $E_{\gamma}=\frac{1}{2} M_{\mathrm{DM}} c^{2}$, we convert the upper bound on the flux limit into the lower limit on decaying DM lifetime:

$$
\begin{aligned}
\tau_{\mathrm{DM}}= & \frac{S_{\mathrm{DM}} \Omega_{\mathrm{fov}}}{8 \pi E_{\gamma} F_{\text {line }}} \\
\approx & 3.7 \times 10^{29} \sec \left(\frac{S_{\mathrm{DM}}}{10^{2} M_{\odot} / \mathrm{pc}^{2}}\right)\left(\frac{\Omega_{\mathrm{fov}}}{1 \mathrm{deg}^{2}}\right) \\
& \times\left(\frac{10 \mathrm{keV}}{E_{\gamma}}\right)\left(\frac{10^{-6} \mathrm{ph} / \mathrm{sec} / \mathrm{cm}^{2}}{F_{\text {line }}}\right)
\end{aligned}
$$

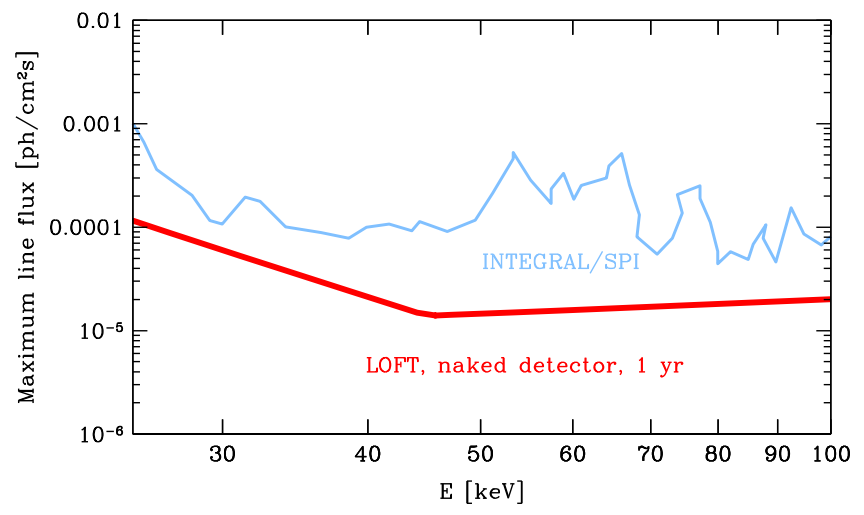

FIG. 6 (color online). Flux limits on DM decay line with large FoV (bare) LAD detector expected from a year-long observation of diffuse emission within $\Omega \simeq 1 \mathrm{sr}$ FoV. For comparison, the limits found from a long (multiyear) exposure of SPI spectrometer on board of INTEGRAL satellite are shown (light blue curve).

(which is about $10^{12}$ times larger than the age of the Universe). From Fig. 5 one sees that the upper limits on the line flux are expected to be at the level of $10^{-6}-10^{-5} \mathrm{ph} / \mathrm{cm}^{2} / \mathrm{sec}$. Substituting these values into (4) one finds the sensitivity of the LAD detector at the level $\tau_{\mathrm{DM}} \sim 10^{29} \mathrm{sec}$, at least an order of magnitude better than existing bounds at these energies. This limit is shown in Fig. 7 as a function of energy. To estimate the sensitivity in the naked detector mode, we assume that the FoV of the detector grows as a power law in the $20-40 \mathrm{keV}$ energy range. Detailed simulations are needed to get a more precise estimate of the opening of the FoV with increasing energy.

\section{IMPLICATIONS FOR STERILE NEUTRINO DM MODELS}

The sterile neutrino is a decaying DM candidate that had recently attracted a lot of attention (see e.g. [49,70-72] for review). Sterile neutrino is a right-chiral counterpart of the ordinary (left-chiral) neutrinos $\nu_{e}, \nu_{\mu}, \nu_{\tau}$. Adding these particles to the SM Lagrangian makes neutrinos massive and provides a simple and elegant explanation for the observed neutrino flavor oscillations and for the smallness of neutrino masses (the so-called "type I seesaw model") [73-76]. These particles are neutral with respect to all standard model interactions (weak, strong and electromagnetic) (see e.g. $[49,77]$ for details). They interact with the matter only via mixing with ordinary neutrinos and in this way effectively participate in weak reactions [49] with strongly suppressed rate (as compared to the ordinary neutrinos). Production of such particles in the primordial plasma [15,78-81] and their decays are controlled by the same parameter-sterile neutrino mixing angle $\sin ^{2}(2 \theta) \lll 1$ inversely proportional to their lifetime: 


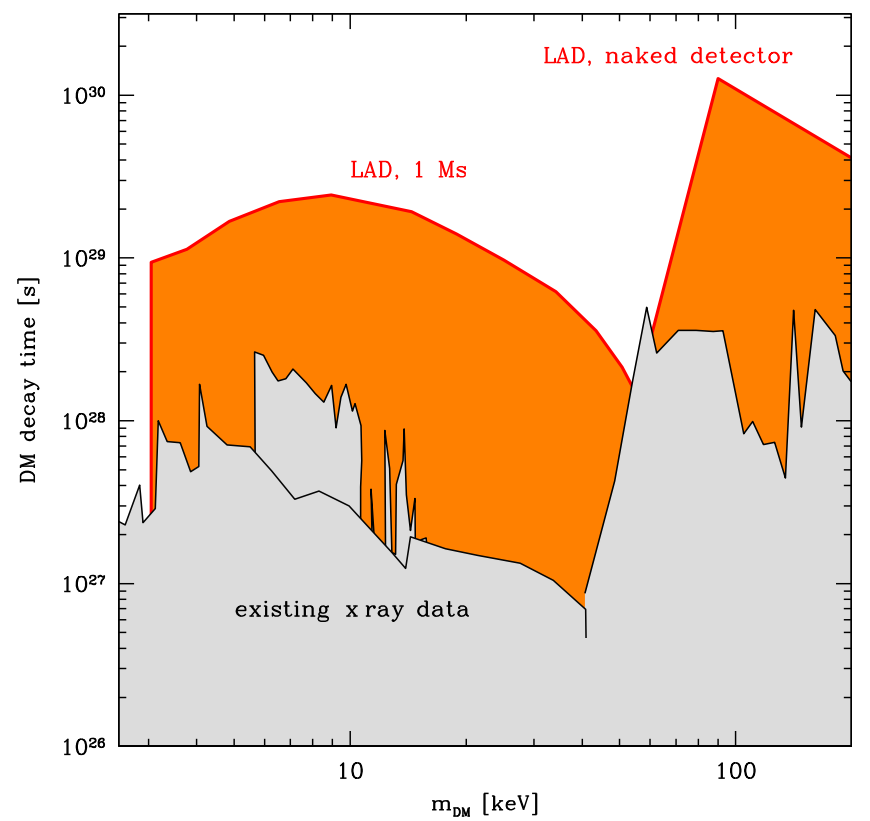

FIG. 7 (color online). Bounds on lifetime of decaying dark matter (for decays DM $\rightarrow \gamma+\nu$ or DM $\rightarrow \gamma+\gamma$ ) (grey shading) and expected improvement from the LOFT LAD detector. The red solid line shows possible LOFT bound assuming $1 \mathrm{Ms}$ exposure with the average dark matter column density $S=300 \mathrm{M}_{\odot} / \mathrm{pc}^{2}$.

$$
\begin{aligned}
\tau_{\mathrm{DM}} & =\frac{1024 \pi^{4}}{9 \alpha G_{F}^{2} \sin ^{2}(2 \theta) m_{\mathrm{DM}}^{5}} \\
& \approx 7.2 \times 10^{29} \sec \left[\frac{10^{-8}}{\sin ^{2}(2 \theta)}\right]\left[\frac{1 \mathrm{keV}}{m_{\mathrm{DM}}}\right]^{5}
\end{aligned}
$$

To be a DM candidate, the interaction strength of sterile neutrinos should be too feeble to make any sizable contribution to active neutrino masses [82].

The 2 MSM model provides an explanation to three known beyond standard model of particle physics phenomena: dark matter, baryon asymmetry of the Universe and neutrino masses, adding three sterile neutrinos to the standard model particle content $[16,83]$. The lightest of the three sterile neutrinos served as the DM. The combination of x-ray bounds of primordial abundance results in both upper and lower bounds on the mass and mixing angle of DM sterile neutrino in the $\nu \mathrm{MSM}$. The range of allowed masses of sterile neutrino DM is $1-50 \mathrm{keV}[44,49,70]$.

The estimates of the bound on the DM sterile neutrino mixing angle expected from LOFT observations are shown in Fig. 8. Interestingly, the requirements configuration of LOFT is expected to provide the best constraints. This is mostly due to the fact that the goal configuration is optimized for point sources and therefore LAD FoV is reduced from $\sim 1^{\circ}$ to $\sim 0.5^{\circ}$. This reduces four times the expected signal from DM decays (provided the DM column density is constant across the FoV) while the background level reduced only slightly.

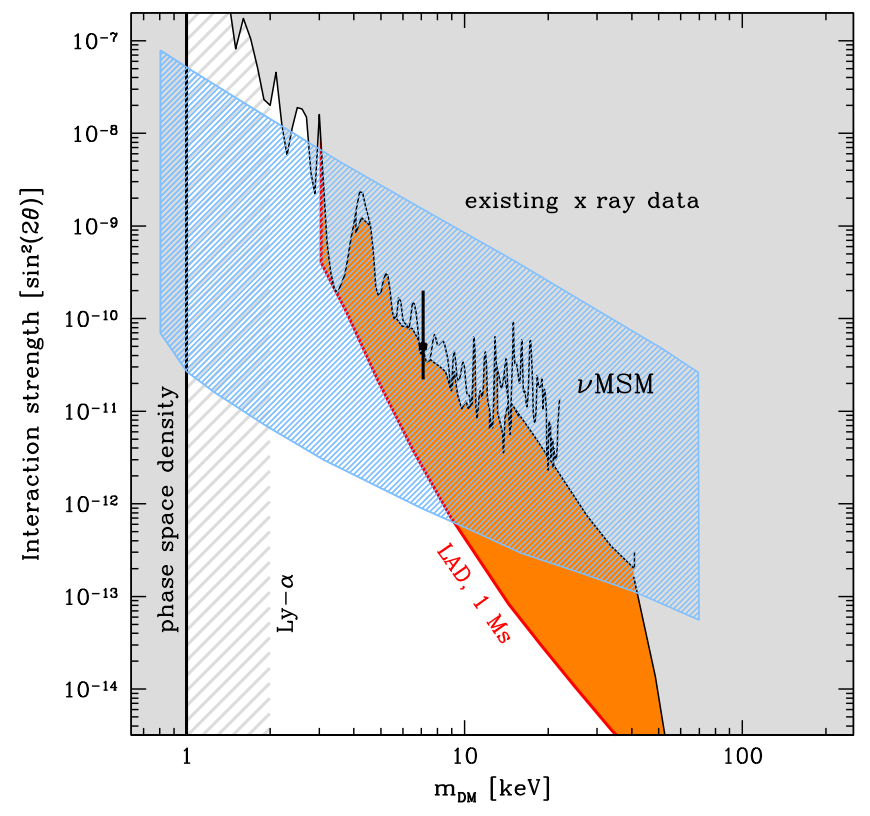

FIG. 8 (color online). Grey shading: Bounds on sterile neutrino parameters [54]. Blue hatching shows the allowed parameter space of the $\nu \mathrm{MSM}$ model. The vertical solid line marks the lower bound on the mass from the phase space density constraint; the grey-hatched region shows the excluded limit based on the Ly $\alpha$ data. The data point with error bars makes the claimed detection of the $7.1 \mathrm{keV}$ decaying dark matter line in galaxy clusters and M31 [58,59]. Orange shading shows the sensitivity limit of LOFT for $1 \mathrm{Ms}$ exposure.

One could see that LOFT will be able to explore a significant fraction of the available range of the mixing angles $\theta$ within $\nu$ MSM. Already one 1 Ms long exposure of a dSph galaxy like Ursa Minor will improve the existing bounds on $\theta$ by two orders of magnitude. Moreover, taking into account importance of the DM nature problem, and the unique characteristics of LOFT, which make it an excellent DM detector, one could imagine a scenario in which the LAD instrument might be operated as a dedicated DM detector (e.g. toward the end of the mission), accumulating a total year scale exposure of a nearby DM halo. This would allow a further boost of sensitivity of the detector by an order of magnitude. In this case LOFT will provide an almost full test of the $\nu \mathrm{MSM}$ and either discover the sterile neutrinos or possibly leave only a narrow window of mass $1 \mathrm{keV}<m_{w d m}<4 \mathrm{keV}$, where the Ly- $\alpha$ bound suffers from some uncertainties [70], unexplored. To probe the mass range below $4 \mathrm{keV}$, one might use the LAD data in the energy range below $2 \mathrm{keV}$. It is clear that the quality of the data in this range is significantly degraded. However, taking into account the unique possibility to explore the full allowed parameter space of a viable DM model (to find the DM or rule out the model) might serve as a good motivation for the challenging task of data analysis in this energy range. 


\section{CONCLUSIONS}

We have shown that LOFT will be a powerful detector of light decaying DM. From Figs. 7 and 8 it is clear that LOFT will be one to two orders of magnitude more sensitive for the detection of DM line in the DM mass range 4-200 keV than all ongoing and past missions. This will provide a qualitatively new insight into the nature of the DM particles within various $\Lambda \mathrm{WDM}$ scenarios, including the most popular one with sterile neutrino DM. Significant improvement is also expected at the highest energies above $30 \mathrm{keV}$, where the LAD instrument becomes a naked detector with the steradian-scale FoV. Such a configuration proves to be optimal for a search of the diffuse all-sky signal from DM decaying in the Milky Way halo (c.f. $[45,61])$.

The energy range of LOFT is crucially important for testing the reference $\iota \mathrm{MSM}$ model. This is clear from Fig. 8. If operated as a dedicated DM search experiment, LOFT will be able to probe almost all the parameter space of the $\nu$ MSM.
[1] S. Tremaine and J. E. Gunn, Phys. Rev. Lett. 42, 407 (1979).

[2] J. Madsen, Phys. Rev. Lett. 64, 2744 (1990).

[3] J. Madsen, Phys. Rev. D 44, 999 (1991).

[4] A. Boyarsky and O. Ruchayskiy, in Proceedings of 4th Patras Workshop on Axions, WIMPs and WISPs, DESY, Hamburg, Germany, 2008 (Verlag Deutsches ElektronenSynchrotron, Hamburg, 2008).

[5] G. Bertone, W. Buchmuller, L. Covi, and A. Ibarra, J. Cosmol. Astropart. Phys. 11 (2007) 003.

[6] R. Essig, E. Kuflik, S. D. McDermott, T. Volansky, and K. M. Zurek, J. High Energy Phys. 11 (2013) 193.

[7] A. Boyarsky, J. Lesgourgues, O. Ruchayskiy, and M. Viel, J. Cosmol. Astropart. Phys. 05 (2009) 012.

[8] M. Cirelli, Pramana J. Phys. 79, 1021 (2012).

[9] S. Profumo, arXiv:1301.0952.

[10] G. Bertone, D. Hooper, and J. Silk, Phys. Rep. 405, 279 (2005).

[11] J. L. Feng, Annu. Rev. Astron. Astrophys. 48, 495 (2010).

[12] L. E. Strigari, Phys. Rep. 531, 1 (2013).

[13] A. e. a. Abramowski, Phys. Rev. Lett. 110, 041301 (2013).

[14] Fermi-LAT Collaboration, Phys. Rev. D 88, 082002 (2013).

[15] S. Dodelson and L. M. Widrow, Phys. Rev. Lett. 72, 17 (1994).

[16] T. Asaka, S. Blanchet, and M. Shaposhnikov, Phys. Lett. B 631, 151 (2005).

[17] M. Lattanzi and J. W. F. Valle, Phys. Rev. Lett. 99, 121301 (2007).

[18] J. P. Conlon and F. Quevedo, J. Cosmol. Astropart. Phys. 08 (2007) 019.

[19] F. Takayama and M. Yamaguchi, Phys. Lett. B 485, 388 (2000).

[20] W. Buchmuller, L. Covi, K. Hamaguchi, A. Ibarra, and T. Yanagida, J. High Energy Phys. 03 (2007) 037.

[21] P. Sikivie, Lect. Notes Phys. 741, 19 (2008).

[22] M. Kawasaki and K. Nakayama, Annu. Rev. Nucl. Part. Sci. 63, 69 (2013).

[23] L. Covi, H.-B. Kim, J. E. Kim, and L. Roszkowski, J. High Energy Phys. 05 (2001) 033.

[24] K.-Y. Choi, J. E. Kim, and L. Roszkowski, J. Korean Phys. Soc. 63, 1685 (2013).

[25] M. Taoso, G. Bertone, and A. Masiero, J. Cosmol. Astropart. Phys. 03 (2008) 022.
[26] F. D. Steffen, Eur. Phys. J. C 59, 557 (2009).

[27] A. Boyarsky, A. Neronov, O. Ruchayskiy, and M. Shaposhnikov, Mon. Not. R. Astron. Soc. 370, 213 (2006).

[28] A. Boyarsky, A. Neronov, O. Ruchayskiy, and M. Shaposhnikov, Phys. Rev. D 74, 103506 (2006).

[29] A. Boyarsky, A. Neronov, O. Ruchayskiy, M. Shaposhnikov, and I. Tkachev, Phys. Rev. Lett. 97, 261302 (2006).

[30] C. R. Watson, J. F. Beacom, H. Yuksel, and T. P. Walker, Phys. Rev. D 74, 033009 (2006).

[31] A. Boyarsky, J. Nevalainen, and O. Ruchayskiy, Astron. Astrophys. 471, 51 (2007).

[32] A. Boyarsky, D. Iakubovskyi, O. Ruchayskiy, and V. Savchenko, Mon. Not. R. Astron. Soc. 387, 1361 (2008).

[33] M. Loewenstein and A. Kusenko, Astrophys. J. 751, 82 (2012).

[34] S. Riemer-Sørensen, S. H. Hansen, and K. Pedersen, Astrophys. J. 644, L33 (2006).

[35] S. Riemer-Sørensen, K. Pedersen, S. H. Hansen, and H. Dahle, Phys. Rev. D 76, 043524 (2007).

[36] A. Boyarsky, O. Ruchayskiy, and M. Markevitch, Astrophys. J. 673, 752 (2008).

[37] S. Riemer-Sørensen and S. H. Hansen, Astron. Astrophys. 500, L37 (2009).

[38] M. Loewenstein and A. Kusenko, Astrophys. J. 714, 652 (2010).

[39] A. Boyarsky, O. Ruchayskiy, M. G. Walker, S. Riemer-Sørensen, and S. H. Hansen, Mon. Not. R. Astron. Soc. 407, 1188 (2010).

[40] M. Loewenstein, A. Kusenko, and P. L. Biermann, Astrophys. J. 700, 426 (2009).

[41] A. Kusenko, M. Loewenstein, and T. T. Yanagida, Phys. Rev. D 87, 043508 (2013).

[42] N. Mirabal, Mon. Not. R. Astron. Soc. 409, L128 (2010).

[43] H. Yuksel, J. F. Beacom, and C. R. Watson, Phys. Rev. Lett. 101, 121301 (2008).

[44] A. Boyarsky, D. Malyshev, A. Neronov, and O. Ruchayskiy, Mon. Not. R. Astron. Soc. 387, 1345 (2008).

[45] A. Boyarsky, J. W. den Herder, A. Neronov, and O. Ruchayskiy, Astropart. Phys. 28, 303 (2007).

[46] K. N. Abazajian, M. Markevitch, S. M. Koushiappas, and R. C. Hickox, Phys. Rev. D 75, 063511 (2007). 
[47] D. A. Prokhorov and J. Silk, arXiv:1001.0215 [Phys. Rev. Lett. (to be published)].

[48] K. Koyama, Y. Hyodo, T. Inui, H. Nakajima, H. Matsumoto, T. G. Tsuru, T. Takahashi, Y. Maeda, N. Y. Yamazaki, H. Murakami et al., Publ. Astron. Soc. Jpn. 59, S245 (2007).

[49] A. Boyarsky, D. Iakubovskyi, and O. Ruchayskiy, Phys. Dark Univ. 1, 136 (2012).

[50] N. Mirabal and D. Nieto, arXiv:1003.3745.

[51] E. Borriello, M. Paolillo, G. Miele, G. Longo, and R. Owen, Mon. Not. R. Astron. Soc. 425, 1628 (2012).

[52] C. R. Watson, Z. Li, and N. K. Polley, J. Cosmol. Astropart. Phys. 3 (2012) 018.

[53] S. Horiuchi, P. J. Humphrey, J. Oñorbe, K. N. Abazajian, M. Kaplinghat, and S. Garrison-Kimmel, Phys. Rev. D 89, 025017 (2014).

[54] D. Malyshev, A. Neronov, and D. Eckert, Phys. Rev. D 90, 103506 (2014).

[55] http://www.isdc.unige.ch/loft.

[56] A. Boyarsky, O. Ruchayskiy, D. Iakubovskyi, A. V. Maccio', and D. Malyshev, arXiv:0911.1774.

[57] A. Boyarsky, A. Neronov, O. Ruchayskiy, and I. Tkachev, Phys. Rev. Lett. 104, 191301 (2010).

[58] E. Bulbul, M. Markevitch, A. Foster, R. K. Smith, M. Loewenstein, and S.W. Randall, Astrophys. J. 789, 13 (2014).

[59] A. Boyarsky, O. Ruchayskiy, D. Iakubovskyi, and J. Franse, arXiv:1402.4119.

[60] K. N. Abazajian, arXiv:0903.2040 [The Astro 2010 Decadal Survey, Cosmology and Fundamental Physics Science (to be published)].

[61] A. Boyarsky, J. W. den Herder, O. Ruchayskiy et al., arXiv:0906.1788.

[62] E. Churazov, R. Sunyaev, M. Revnivtsev, S. Sazonov, S. Molkov, S. Grebenev, C. Winkler, A. Parmar, A. Bazzano, M. Falanga et al., Astron. Astrophys. 467, 529 (2007).

[63] J. Kaastra, http://space.mit.edu/home/nss/Jelle_Kaastra_ ixo_spextroscopy.pdf.
[64] M. Feroci, L. Stella, M. van der Klis, T. J.-L. Courvoisier, M. Hernanz, R. Hudec, A. Santangelo, D. Walton, A. Zdziarski, D. Barret et al., Exp. Astron. 34, 415 (2012).

[65] S. Zane, D. Walton, T. Kennedy, M. Feroci, J.-W. Den Herder, M. Ahangarianabhari, A. Argan, P. Azzarello, G. Baldazzi, D. Barret et al., arXiv:1209.1498.

[66] Available at [67].

[67] http://www.isdc.unige.ch/loft/index.php/preliminar-response -files-and-simulated-background.

[68] R. Campana, M. Feroci, E. Del Monte, T. Mineo, N. Lund, and G. W. Fraser, Exp. Astron. 36, 451 (2013).

[69] E. Churazov, S. Sazonov, S. Tsygankov, R. Sunyaev, and D. Varshalovich, Mon. Not. R. Astron. Soc. 411, 1727 (2011).

[70] A. Boyarsky, O. Ruchayskiy, and M. Shaposhnikov, Annu. Rev. Nucl. Part. Sci. 59, 191 (2009).

[71] A. Kusenko, Phys. Rep. 481, 1 (2009).

[72] M. Drewes, Int. J. Mod. Phys. E 22, 1330019 (2013).

[73] P. Minkowski, Phys. Lett. B 67, 421 (1977).

[74] P. Ramond, arXiv:hep-ph/9809459.

[75] R. N. Mohapatra and G. Senjanovic, Phys. Rev. Lett. 44, 912 (1980).

[76] T. Yanagida, Prog. Theor. Phys. 64, 1103 (1980).

[77] K. Abazajian, M. Acero, S. Agarwalla, A. Aguilar-Arevalo, C. Albright et al., arXiv:1204.5379.

[78] X.-d. Shi and G. M. Fuller, Phys. Rev. Lett. 82, 2832 (1999).

[79] K. Abazajian, G. M. Fuller, and M. Patel, Phys. Rev. D 64, 023501 (2001).

[80] T. Asaka, M. Laine, and M. Shaposhnikov, J. High Energy Phys. 06 (2006) 053.

[81] T. Asaka, M. Laine, and M. Shaposhnikov, J. High Energy Phys. 01 (2007) 091.

[82] A. Boyarsky, A. Neronov, O. Ruchayskiy, and M. Shaposhnikov, JETP Lett. 83, 133 (2006).

[83] T. Asaka and M. Shaposhnikov, Phys. Lett. B 620, 17 (2005). 